\title{
Efficacy of Trimetazidine Dihydrochloride for Relieving Chronic Tinnitus: A Randomized Double-Blind Study
}

\author{
Tolgar Lütfi Kumral · GüvenYıldııım · Güler Berkiten · Ziya Saltürk · Enes Ataç · Yavuz Atar · Yavuz Uyar \\ Department of Otorhinolaryngology-Head and Neck Surgery, Okmeydanı Training and Research Hospital, Istanbul, Turkey
}

Objectives. To evaluate the efficacy of trimetazidine dihydrochloride as a treatment for chronic tinnitus.

Methods. A total of 97 chronic tinnitus patients were evaluated in this randomized, prospective, double-blind, placebo-controlled trial. After assessing for eligibility, 82 patients were randomly assigned into placebo or trimetazidine groups according to the medication. The trimetazidine group received $20 \times 3 \mathrm{mg} /$ day per oral trimetazidine dihydrochloride and the placebo group received $20 \times 3 \mathrm{mg} /$ day per oral placebo for 3 months. Tinnitus handicap inventory (THI), visual analogue scale (VAS) questionnaires and audiometric results were used to determine the effectiveness of trimetazidine treatment.

Results. The study group comprised 82 tinnitus subjects, 42 (51\%) of whom received trimetazidine dihydrochloride and 40 $(49 \%)$ who received placebo. There was no significant difference between placebo and trimetazidine groups in THI grade and VAS (both pre- and posttreatment scores) $(P>0.05)$ and no significant improvement was observed in subjective loudness score in either group $(P>0.05)$. Additionally there was no significant difference between groups in pre- and posttreatment pure tone hearing thresholds at all measured frequencies $(P>0.05)$.

Conclusion. Trimetazidine dihydrochloride therapy was ineffective for relieving chronic tinnitus.

Keywords. Tinnitus; Trimetazidine Dihydrochloride; Drug Treatment

\section{INTRODUCTION}

Subjective tinnitus is defined as the perception of a sound in the absence of an external stimulus [1]. Tinnitus is a common complaint that can affect a patient's quality of life. The prevalence of tinnitus has been variously reported as ranging $2 \%-32 \%[1,2]$. Although the pathological mechanisms and clinical features are still not fully understood, there is growing evidence that changes

\footnotetext{
- Received April 21, 2015

Revised July 5, 2015

Accepted July 6, 2015

- Corresponding author: Tolgar Lütfi Kumral

Department of Otorhinolaryngology-Head and Neck Surgery, Okmeydanı

Training and Research Hospital, Darülaceze Cad. No:25 posta kodu: 34400 Okmeydanı, Sişli, Istanbul, Turkey

Tel: +90-212-314-55-55, Fax: +90-212-221-78-00

E-mail: tolgins@hotmail.com
}

in neuronal activity in different parts of the auditory pathway, including the dorsal cochlear nucleus, inferior colliculus, thalamus and/or auditory cortex underlie tinnitus pathology [3]. Tinnitus can be associated with virtually all disorders affecting the auditory system and can be accompanied by hearing loss in many cases [4]. Reduced auditory input from a partly deafferented cochlea in the central nervous system can lead to tinnitus. On the other hand, hearing loss is not always a mandatory requirement for some patients' tinnitus generation.

The treatment of chronic tinnitus is very troublesome and there is no single treatment in terms of imparting replicable long-term improvement of tinnitus impact [5]. Since the pathophysiology of tinnitus is largely unknown, treatment plans have focused on symptomatic relief. However, there is no universally accepted standard of care for tinnitus or approved tinnitus drug.

A large variety of pharmacological agents are used for the

Copyright (C) 2016 by Korean Society of Otorhinolaryngology-Head and Neck Surgery.

This is an open-access article distributed under the terms of the Creative Commons Attribution Non-Commercial License (http://creativecommons.org/licenses/by-nc/4.0)

which permits unrestricted non-commercial use, distribution, and reproduction in any medium, provided the original work is properly cited. 
treatment of tinnitus and the results are quite variable. Some medications are prescribed to relieve the associated symptoms of the tinnitus such as depression, anxiety or insominia whereas some of them are applied for changing the pathophysiology. Lidocaine, benzodiazepines, antidepressants, anticonvulsants, antiglutamatergic agents target to the neural activity underlying tinnitus. Others such as systemic and intratympanic steroids, ginkgo biloba, and melatonin have anti-inflammatory, vasodilator or antioxidant effects on cochlea and neural pathways [1,5].

There are only few studies about the efficacy of trimetazidine dihydrochloride on vertigo and tinnitus [6,7]. Trimetazidine is a cytoprotective anti-ischemic agent. Trimetazidine controls oxidative stress, preserves mitochondrial respiration in ischemia-reperfusion injury, and prevents cardiac ischemia [8].

The fields of use of trimetazidine in otorhinolaryngology include the reduction in vertigo duration and frequency, treatment of Meniere's disease related cochleovestibular conditions, correction of inner ear ischemia, correction of isolated tinnitus $[6,7,9,10]$.

Trimetazidine dihydrochloride is a commonly used drug in the treatment of tinnitus nowadays. However, the efficacy for relieving symptoms is controversial. The purpose of this study was to investigate the efficacy of trimetazidine dihydrochloride therapy for relieving chronic tinnitus.

\section{MATERIALS AND METHODS}

Between January 2013 and December 2013, a total of 97 tinnitus patients were assessed for eligibility. Institutional Review Board approval was taken from the Ethical Committee of Okmeydanı Training and Research Hospital. Informed consents of all participants were obtained following a detailed explanation of all probable benefits and risks of the therapy.

This study was designed as a randomized, prospective, doubleblind trial. Primary complaint of continuous, chronic subjective tinnitus for at least 6 months, age over 18 without any neurologic and systemic diseases were the inclusion criteria in this study.

All of the patients underwent complete otolaryngological examination. Patients who had tympanic membrane perforation, otitis media with effusion, otosclerosis, tympanosclerosis, chron-

\section{H I G H L I G G H T S}

- The efficacy of trimetazidine dihydrochloride for chronic tinnitus was prospectively assessed in 97 patients.

- Tinnitus and audiometric results between placebo and trimetazidine groups were compared in a randomized, doubled-blind trial.

- The randomized controlled trial failed to show any statistical differences between two groups. ic otitis media, sudden hearing loss, any otologic surgery, chronic drug users for a specific disease, systemic illness like hyperlipidemia, hypertension, and hyperthyroidism were excluded from the study.

Of these 97 patients, 9 patients were excluded for not meeting eligibility criteria. Eighty-eight patients were randomly assigned into 2 groups according to treatment. Of the 88 patients included, 6 patients ( 2 in trimetazidine group and 4 in the placebo group) missed the 3-month follow-up visit. Overall, 82 patients (42 trimetazidine group, 40 placebo group) who completed the 3-month treatment intervention were included in the perprotocol analysis.

Patients underwent pure tone audiometry in order to compare hearing thresholds at standard frequencies and high frequencies. All of the audiometric evaluations were performed using a calibrated Interacustic AC-40 Audiometry (Interacoustics, Assens, Denmark) in a soundproof cabin with an ISO 9001 standards. Frequencies between 250 and $20,000 \mathrm{~Hz}$ were tested for air and bone conduction at frequencies between 500 and $4,000 \mathrm{~Hz}$. Audiometric threshold was considered as the pure tone average for the frequencies $0.5-1-2-4-8 \mathrm{kHz}$ and assessed as normal below $40 \mathrm{~dB}$. The mean hearing threshold levels in both ears of patients were compared after 3 months between the groups.

In this randomized, double-blind study, the subjects were given 3-month supply of either $20 \times 3 \mathrm{mg} /$ day per oral capsules of trimetazidine or placebo. This regimen was chosen because it is the general recommended dose. Trimetazidine and placebo capsules were dispensed by an independent pharmacist. Each capsule contains $20 \mathrm{mg}$ of trimetazidine dihydrochloride. The placebo capsules contained sugar powder and were identical in color and smell with the trimetazidine capsules. Patients were randomly assigned into 2 groups by a computer-based research randomization program. The code remained unknown to the subjects, physicians, and staff members. Another physician kept the randomization code.

The study group consisted of 42 patients ( 25 females and 17 males) and received $20 \times 3 \mathrm{mg} /$ day per oral trimetazidine dihydrochloride. The placebo group consisted of 40 patients (22 female and 18 male) and received $20 \times 3 \mathrm{mg} /$ day per oral matchedplacebo (capsules with sugar powder). None of the patients had drug intolerance or side effects.

\section{Questionnaire}

A survey form was completed before treatment. All patients were required to answer 2 sets of questionnaires. Tinnitus handicap inventory (THI) and visual analogue scale (VAS) questionnaires were used to determine the effectiveness of trimetazidine treatment.

The VAS questionnaires are symptom rating scales that require the patient to assign a 1-10 score based on the perceived loudness. Patients rated the loudness of their tinnitus on a scale 
ranging from 1 (very quiet) to 10 (very loud).

THI is 25-item tool developed by Newman et al. [11] and designed to measure effects of tinnitus on daily life. Each question was numbered as 0,2 , and 4 . Zero (0) represented no problem, 4 represented severe problem, and 2 represented sometimes. Results are graded as $0-16$, slight or no handicap (grade 1); 1836 , mild handicap (grade 2); 38-56, moderate handicap (grade 3); 58-76, severe handicap (grade 4); 78-100, catastrophic handicap (grade 5) [12].

Tinnitus questionnaires were also completed by the patients after 3 months of treatment and compared between the groups.

\section{Statistical analysis}

In the statistical model, sex (male/female), age group, VAS, THI and audiometric results were evaluated as the main factors. For statistical analysis, SPSS ver. 17.0 (SPSS Inc., Chicago, IL, USA) was used to assess the findings of the study. The normal distributions of the parameters were evaluated by Shapiro-Wilks test and parameters were found to be normally distributed. Descriptive statistical methods (mean, standard deviation), as well as paired sample $t$-test and Student $t$-test for the comparison of quantitative data showing the parameters of the normal distribution and chi-square test of qualitative data were used for the determination of difference between the groups. The significance level were set as $P<0.05$ and $95 \%$ confidence interval.

\section{RESULTS}

The study group recruited herein comprised 82 tinnitus subjects, $42(51 \%)$ of whom received trimetazidine dihydrochloride and $40(49 \%)$ who received placebo. The study population consisted of 35 male and 47 female subjects. All of the patients had experienced tinnitus for between 6 months and 10 years. A total of 46 patients $(56 \%)$ consistently reported that their tinnitus was localized to one ear; the remaining 36 patients (44\%) experienced tinnitus in both ears. In terms of type of noise, patients reported experiencing hissing $(n=25)$, ringing $(n=22)$, and tonal $(\mathrm{n}=35)$ tinnitus. Tinnitus was constant in 54 subjects $(66 \%)$, whereas intermittent in 28 subjects (34\%). The pretreatment data for each group are provided in Table 1.

There were no significant differences between the 2 treatment groups with regard to age, sex, mean pure tone hearing thresholds, pretreatment VAS for loudness and THI scores, tinnitus laterality or duration $(P>0.05)$ (Table 1$)$.

Mean pretreatment THI scores (35.1 and 34.8 in the trimetazidine and placebo groups, respectively) did not differ between groups $(P=0.866)$, both of which experienced improved scores posttreatment $(P=0.001, P=0.013)$. However, the mean posttreatment THI scores was not significantly different between the groups $(P=0.234)$ (Table 2$)$.

There was also no significant difference between groups in mean pre- or posttreatment THI grade $(P>0.05)$; THI grade did

Table 1. Baseline characteristics

\begin{tabular}{lcc}
\hline Characteristic & Trimetazidine group & Placebo group \\
\hline Sex (male:female) & $17: 25$ & $18: 22$ \\
Age (yr) & $41 \pm 10$ & $45 \pm 9$ \\
Tinnitus type & & 0.679 \\
$\quad$ Hissing:ringing:total & $15: 8: 19$ & $10: 14: 16$ \\
Tinnitus laterality & & 0.088 \\
$\quad$ Unilateral:bilateral & $25: 17$ & $21: 19$ \\
Pure tone average (dB HL) & & $24.8 \pm 3.0$ \\
$\quad$ Right & $26.1 \pm 3.7$ & $25.3 \pm 3.1$ \\
$\quad$ Left & $26.6 \pm 3.1$ & $5.6 \pm 1.6$ \\
TI duration (yr) & $6.4 \pm 2.2$ & $34.8 \pm 9.1$ \\
Global THI & $35.1 \pm 9.2$ & 0.072 \\
Tinnitus loudness VAS (0-10) & $5.1 \pm 1.2$ & 0.059 \\
\hline
\end{tabular}

Values are presented as mean $\pm \mathrm{SD}$.

$\mathrm{HL}$, hearing loss; TI, tinnitus; THI, tinnitus handicap inventory; VAS, visual analogue scale.

$\mathrm{TH}=$ range $0-100, \mathrm{VAS}=$ range $0-10$. Pure tone average $(500,1,000,2,000,4,000,8,000 \mathrm{~Hz}$ mean $)$.

Table 2. Evaluation of the mean pretreatment and posttreatment THI scores

\begin{tabular}{|c|c|c|c|}
\hline Group & Pretreatment & Posttreatment & First-last change $\left(P\right.$-value $\left.{ }^{\mathrm{b})}\right)$ \\
\hline Trimetazidine $(n=42)$ & $35.1 \pm 9.2$ & $29.9 \pm 8.2$ & 0.001 \\
\hline Placebo $(n=40)$ & $34.8 \pm 9.1$ & $32.3 \pm 9.9$ & 0.013 \\
\hline$P$-value ${ }^{\text {a) }}$ & 0.866 & 0.234 & - \\
\hline
\end{tabular}

Values are presented as mean \pm SD.

$\mathrm{THI}$, tinnitus handicap inventory.

a)Independent samples test. ${ }^{\text {b) }}$ Paired sample $t$-test. $P>0.05$. 
Table 3. Evaluation of the mean pretreatment and posttreatment THI grading

\begin{tabular}{lccc}
\hline Group & Pretreatment & Posttreatment & First-last change $\left(P\right.$-value $^{\text {b) }}$ \\
\hline Trimetazidine $(n=42)$ & $2.4 \pm 0.7$ & $2.2 \pm 0.6$ & 0.110 \\
Placebo $(n=40)_{P}$-value $^{\text {a }}$ & $2.3 \pm 0.6$ & $2.3 \pm 0.7$ & 0.486 \\
\hline
\end{tabular}

Values are presented as mean $\pm \mathrm{SD}$.

$\mathrm{TH}$, tinnitus handicap inventory.

a)Independent samples test. ${ }^{\mathrm{b})}$ Paired sample $t$-test. $P>0.05$.

Table 4. Improvement in tinnitus handicap inventory grading

\begin{tabular}{lcccc}
\hline \multirow{2}{*}{ Group } & & THl grading change & Worsen & \multirow{2}{*}{ Total } \\
\cline { 2 - 4 } & Improved & No improvement & $2(5)$ & 42 \\
\hline Trimetazidine & $11(26)$ & $29(69)$ & $3(8)$ & 40 \\
Placebo & $6(15)$ & $31(78)$ & $5(6)$ & 82 \\
Total & $17(21)$ & $60(73)$ & \\
\hline
\end{tabular}

Values are presented as number (\%).

Pearson chi-square $=1.689 . \quad P=0.430$.

Table 5. Evaluation of the pretreatment and posttreatment visual analogue scale scores for loudness

\begin{tabular}{lccc}
\hline Group & Pretreatment & Posttreatment & First-last change $\left(P\right.$-value $\left.^{\text {b) }}\right)$ \\
\hline Trimetazidine $(n=42)$ & $5.1 \pm 1.2$ & $4.9 \pm 1.1$ & 0.272 \\
Placebo $(n=40)_{P_{\text {-value }} \text { a) }}$ & $5.2 \pm 1.2$ & $4.7 \pm 1.2$ & 0.074 \\
\hline
\end{tabular}

Values are presented as mean $\pm \mathrm{SD}$.

a)Independent samples test. ${ }^{b}$ Paired sample $t$-test. $P>0.05$.

Table 6. Improvement in VAS scores for loudness

\begin{tabular}{|c|c|c|c|c|}
\hline \multirow{2}{*}{ Group } & \multicolumn{3}{|c|}{ VAS (loudness) change } & \multirow{2}{*}{ Tota } \\
\hline & Improved & No improvement & Worsen & \\
\hline Trimetazidine & $15(36)$ & $23(55)$ & $4(10)$ & 42 \\
\hline Placebo & $11(28)$ & $22(55)$ & $7(18)$ & 40 \\
\hline Total & $26(32)$ & $45(55)$ & $11(13)$ & 82 \\
\hline
\end{tabular}

Values are presented as number (\%).

VAS, visual analogue scale.

Pearson chi-square $=1.408 . P=0.495$.

not improve after treatment in either group (Table 3).

In the trimetazidine and placebo groups, 69\% $(n=29)$ and $78 \%(n=31)$ of patients, respectively, exhibited no improvement in posttreatmentTHI grade, butTHI grade improved in $26 \%$ (n= $11)$ and $15 \%(n=6)$ of these subjects. There was no significant difference between groups in THI grade improvement rate, which was $21 \%(n=17)$ across the entire sample $(P>0.05)$ (Table 4$)$.

VAS scores were measured before and after treatment in both groups. There was no significant difference between groups in either pre- or posttreatment VAS score, and no significant improvement in the subjective loudness scores in either group $(P>0.05)$ (Table 5).

In the trimetazidine and placebo groups, $55 \%(n=23)$ and $55 \%(n=22)$ of patients, respectively, exhibited no improvement in posttreatment VAS scores for loudness, but VAS scores improved in $36 \%(n=15)$ and $28 \%(n=11)$ of these subjects. There was no significant difference between groups in VAS score improvement rate, which was $32 \%(n=26)$ across the entire sample $(P>0.05)$ (Table 6).

Furthermore, there were no significant differences between groups in pure-tone hearing thresholds, at all measured frequencies, according to posttreatment pure tone standard and highfrequency pure tone audiometry assessment $(P>0.05)$ (Figs. 1, 2).

\section{DISCUSSION}

Previous studies have demonstrated that the cytoprotective effects of trimetazidine are due to the prevention of injury to neurosensorial tissue that may result from overstimulation of the cochleovestibular system by glutamate, and by the antioxidant effects of this compound [13]. In experimental animal studies, 


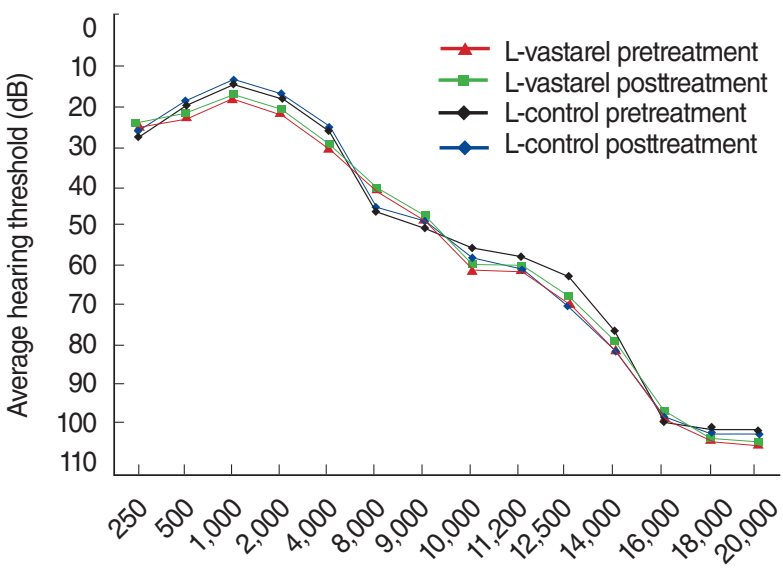

Left ear frequency value $(\mathrm{Hz})$

Fig. 1. Pretreatment and posttreatment hearing levels of tinnitus patients at left ear between 250 and $20,000 \mathrm{~Hz}$.

the ototoxic effect induced by gentamicin and neomycin is prevented by trimetazidine [14]. In otolaryngology, trimetazidine is used to treat vertigo, Meniere disease, and hearing loss due to noise-induced and idiopathic sudden hearing loss $[6,7,9,10]$.

Acute tinnitus is frequently caused by ear conditions or psychosocial stress, whereas chronic tinnitus can begin in response to persistent exposure to noise, whiplash injuries, or functional disorders of the cervical spine [15]. In acute tinnitus, symptoms may improve after treatment of the underlying cause, but treating chronic tinnitus of unknown etiology is highly problematic [16].

Tinnitus could develop associated with most of the disorders affecting the auditory system. For this reason, a variety of pharmacological agents have been tried for the treatment of tinnitus $[5,17,18]$. Most recently, antidepressants and intravenous lidocaine showed a significant decrease in tinnitus severity $[19,20]$. Benzodiazepines, a $\mathrm{GABA}_{\mathrm{A}}$ receptor agonists, are effective in relieving tinnitus. However, adverse side effects such as sedation limit its usage. Baclofen and gabapentin is no more effective than placebo for the relief of idiopathic subjective tinnitus $[21,22]$. Betahistine hydrochloride is used to eliminate tinnitus in patients with vestibular disorders. However, there was insufficient evidence to say whether betahistine has any effect on Menière disease-related tinnitus [23]. There is a growing evidence indicating that gingko is no more effective in alleviating tinnitus symptoms than placebo [24]. Others such as systemic and intratympanic steroids, melatonin, vitamins, zinc, osmotic vasodilators, and regulators have anti-inflammatory, vasodilator or antioxidant effects on cochlea and neural pathways. Systemic and intratympanic steroids have been proposed for the treatment of acute tinnitus related to sudden hearing loss. Osmotic vasodilators and regulators have been used to treat for tinnitus of cochlear origin. Zinc therapy did not result in tinnitus improvement in patients with normal zinc levels. Melatonin was found to improve sleep disturbance linked to tinnitus. The effect of these drugs on the subjective tinnitus is still uncertain due to in-

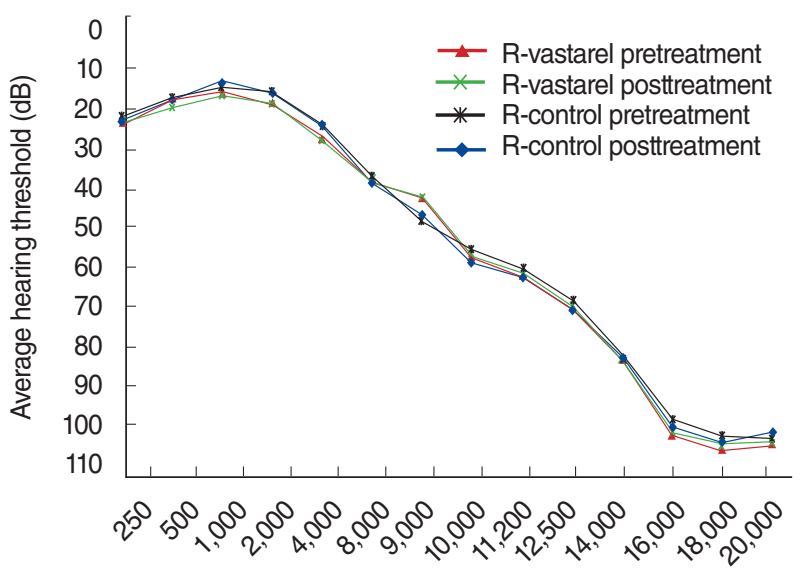

Right ear frequency value $(\mathrm{Hz})$

Fig. 2. Pretreatment and posttreatment hearing levels of tinnitus patients at right ear between 250 and $20,000 \mathrm{~Hz}$.

adequate clinical trials [16-18,21].

Of the few studies that have demonstrated the effectiveness of trimetazidine against tinnitus, the most important study was conducted by Morgon [6]. The efficacy of trimetazidine has not been evaluated by any subsequent study, but the drug is nevertheless commonly prescribed for tinnitus.

THI, VAS questionnaires, and audiometric results were used to determine the effectiveness of trimetazidine treatment. THI scores improved after treatment in both the trimetazidine and placebo groups, but the degree of improvement did not differ between groups, indicating that trimetazidine was no more effective than placebo against tinnitus (Table 2). The improvement in THI scores of the groups was considered as the placebo effect of the both drugs [25].

We classified THI into subgroups per the method employed by McCombe et al. [12]. In total, 17 subjects (21\%) exhibited improvement by one or more grades across both the trimetazidine and placebo groups; 60 subjects $(73 \%)$ remained at the same tinnitus grade posttreatment (Table 4). Mean THI grade did not differ between the 2 groups, again indicating that trimetazidine was no more effective than placebo against tinnitus.

Treatment of subjective tinnitus depends on the patient's perception of its loudness. Tinnitus loudness can be estimated by using loudness matching with an external sound or using direct loudness ratings. Because tinnitus loudness varies widely among patients, and frequently fluctuates within individual patients, quantifying the disability it causes, and devising appropriate treatment plans, is challenging. We usedVAS to assess subjective tinnitus loudness; posttreatment scores did not differ between the 2 groups and pre- and posttreatment change scores were not significant in either group $(P>0.05)$ (Table 5). Across both groups, 26 subjects (32\%) exhibited improvements in VAS, whereas 45 subjects $(55 \%)$ did not $(P>0.05)$ (Table 6). This revealed that the subjective VAS for loudness did not differ in both groups of treatment. 
Tinnitus has a strong association with elderly termed as presbytinnitus. However, this study did not comprise the presbytinnitus. Haguenauer et al. [26] demonstrated the utility of trimetazidine during degenerative hearing loss management. Even if they do not experience subjective hearing impairment, the majority of tinnitus patients exhibit high-frequency hearing loss $[27,28]$. Therefore, we also evaluated high-frequency audiometry results before and after trimetazidine treatment, including in subjects characterized by normal hearing thresholds at higher frequencies. There was no significant difference between the study and placebo groups at any frequency, according to posttreatment standard and high-frequency puretone audiometry assessments; trimetazidine dihydrochloride treatment had no effect on hearing thresholds $(P>0.05)$ (Figs. 1, 2).

In conclusion, trimetazidine dihydrochloride therapy was ineffective for relieving chronic tinnitus.

\section{CONFLICT OF INTEREST}

No potential conflict of interest relevant to this article was reported.

\section{REFERENCES}

1. Jastreboff PJ. Phantom auditory perception (tinnitus): mechanisms of generation and perception. Neurosci Res. 1990 Aug;8(4):221-54.

2. Coles RR. Epidemiology of tinnitus: (1) prevalence. J Laryngol Otol Suppl. 1984;9:7-15.

3. Mattox DE. Assessment and management of tinnitus and hearing loss. Contin: Lifelong Learn Neurol. 2006 Aug;12(4):135-50

4. Konig O, Schaette R, Kempter R, Gross M. Course of hearing loss and occurrence of tinnitus. Hear Res. 2006 Nov;221(1-2):59-64.

5. Dobie RA. A review of randomized clinical trials in tinnitus. Laryngoscope. 1999 Aug;109(8):1202-11.

6. Morgon A. A multicenter double blind versus placebo study of trimetazidine in tinnitus: results obtained after 2 months of treatment. Ann Otolaryngol Chir Cervicofac. 1990;107 Suppl 1:77-81.

7. Martini A, De Domenico F. Trimetazidine versus betahistine in Ménière's disease. a double blind method. Ann Otolaryngol Chir Cervicofac. 1990;107 Suppl 1:20-7.

8. Peng S, Zhao M,Wan J, Fang Q, Fang D, Li K. The efficacy of trimetazidine on stable angina pectoris: a meta-analysis of randomized clinical trials. Int J Cardiol. 2014 Dec;177(3):780-5.

9. Kluyskens P, Lambert P, D'Hooge D. Trimetazidine versus betahistine in vestibular vertigo. A double blind study. Ann Otolaryngol Chir Cervicofac. 1990;107 Suppl 1:11-9.
10. Wayoff M. Evaluation of the therapeutic efficacy of vastarel $20 \mathrm{mg}$ (trimetazidine) in cochleovestibular syndromes. A double-blind study versus placebo. Ann Otolaryngol Chir Cervicofac. 1984;101 (7):565-9.

11. Newman CW, Jacobson GP, Spitzer JB. Development of the tinnitus handicap inventory. Arch Otolaryngol Head Neck Surg. 1996 Feb; 122(2):143-8.

12. McCombe A, Baguley D, Coles R, McKenna L, McKinney C, WindleTaylor P, et al. Guidelines for the grading of tinnitus severity: the results of a working group commissioned by the British Association of Otolaryngologists, Head and Neck Surgeons, 1999. Clin OtolaryngolAllied Sci. 2001 Oct;26(5):388-93.

13. Unal OF, Ghoreishi SM, Atas A, Akyurek N, Akyol G, Gursel B. Prevention of gentamicin induced ototoxicity by trimetazidine in animal model. Int J Pediatr Otorhinolaryngol. 2005 Feb;69(2):193-9.

14. Aksoy F, Dogan R, Ozturan O, Eren SB, Veyseller B, Pektas A, et al. Protective effect of trimetazidine on amikacin-induced ototoxicity in rats. Int J Pediatr Otorhinolaryngol. 2014 Apr;78(4):663-9.

15. Kiani F, Yoganantha U,Tan CM, Meddis R, Schaette R. Off-frequency listening in subjects with chronic tinnitus. Hear Res. 2013 Dec;306: 1-10.

16. Rah YC, Park KT, Yi YJ, Seok J, Kang SI, Kim YH. Successful treatment of sudden sensorineural hearing loss assures improvement of accompanying tinnitus. Laryngoscope. 2015 Jun;125(6):1433-7.

17. Langguth B, Elgoyhen AB. Emerging pharmacotherapy of tinnitus. Expert Opin Emerg Drugs. 2011 Dec;16(4):603-6.

18. Darlington CL, Smith PF. Drug treatments for tinnitus. Prog Brain Res. 2007;166:249-62.

19. Robinson SK, Viirre ES, Stein MB. Antidepressant therapy in tinnitus. Hear Res. 2007 Apr;226(1-2):221-31.

20. Kalcioglu MT, Bayindir T, Erdem T, Ozturan O. Objective evaluation of the effects of intravenous lidocaine on tinnitus. Hear Res. 2005 Jan;199(1-2):81-8.

21. Simpson JJ, Davies WE. Recent advances in the pharmacological treatment of tinnitus. Trends Pharmacol Sci. 1999 Jan;20(1):12-8.

22. Aazh H, El Refaie A, Humphriss R. Gabapentin for tinnitus: a systematic review. Am J Audiol. 2011 Dec;20(2):151-8.

23. James AL, Burton MJ. Betahistine for Menière's disease or syndrome. Cochrane Database Syst Rev. 2001;(1):CD001873.

24. Hilton MP, Zimmermann EF, Hunt WT. Ginkgo biloba for tinnitus. Cochrane Database Syst Rev. 2013 Mar;3:CD003852.

25. Ernst E. Placebo: new insights into an old enigma. Drug Discov Today. 2007 May;12(9-10):413-8.

26. Haguenauer JP, Bebear JP, Bordes LR, Jacquot M, Mercier J, Morgon A, et al. Trimetazidine and degenerative deafness. Effect on hearing and integration. Ann Otolaryngol Chir Cervicofac. 1990;107 Suppl 1:51-6.

27. Kim DK, Park SN, Kim HM, Son HR, Kim NG, Park KH, et al. Prevalence and significance of high-frequency hearing loss in subjectively normal-hearing patients with tinnitus. Ann Otol Rhinol Laryngol. 2011 Aug;120(8):523-8.

28. Shim HJ, Kim SK, Park CH, Lee SH, Yoon SW, Ki AR, et al. Hearing abilities at ultra-high frequency in patients with tinnitus. Clin Exp Otorhinolaryngol. 2009 Dec;2(4):169-74. 